\title{
Signal and zero padding to improve parameters estimation of sinusoidal signals in the frequency domain
}

\author{
Dušan Agrež ${ }^{1}$, Damir Ilić ${ }^{2}$ Janko Drnovšek ${ }^{1}$ \\ ${ }^{1}$ University of Ljubljana, Faculty of Electrical Engineering, Trzaska 25, 1000 Ljubljana, Slovenia \\ ${ }^{2}$ University of Zagreb, Faculty of Electrical Engineering and Computing, Unska 3, 10000 Zagreb, Croatia
}

\begin{abstract}
This paper presents the procedure to improve the estimation of the basic sinusoidal signal parameters (frequency, amplitude, and phase, respectively) in the case of signal sampling by averaging in the aperture time. Prior to estimation in the frequency domain by the interpolated DFT algorithms the sampled signal is padded with the signal average values in the aperture times and zeroes in the rest of the sampling interval. We can increase padding points and a number of the signal cycles in the whole measurement interval and with this nearing the errors to the level as with estimation of the signal without average sampling even the sampling Nyquist condition is not fulfill.
\end{abstract}

Section: RESEARCH PAPER

Keywords: sampling process; sampling by averaging; signal padding; zero padding; estimation of parameters; interpolated DFT

Citation: Dušan Agrež, Damir llić, Janko Drnovšek, Signal and zero padding to improve parameters estimation of sinusoidal signals in the frequency domain, Acta IMEKO, vol. 5, no. 3, article 8, November 2016, identifier: IMEKO-ACTA-05 (2016)-03-08

Section Editor: Konrad Jedrzejewski, Warsaw University of Technology, Poland

Received May 13, 2016; In final form July 11, 2016; Published November 2016

Copyright: (C) 2016 IMEKO. This is an open-access article distributed under the terms of the Creative Commons Attribution 3.0 License, which permits unrestricted use, distribution, and reproduction in any medium, provided the original author and source are credited

Corresponding author: Dušan Agrež, e-mail: dusan.agrez@fe.uni-lj.si

\section{INTRODUCTION}

In the last decade, considerable research has been carried out on the analysis of efficient methods capable of accurately estimating parameters of the frequency components of interest [1], [2]. In many cases the problem of evaluating the spectral performance of a given periodic signal reduces to the estimation of parameters of each spectral component (frequency, amplitude, and phase). Parameter's estimations of periodic signals mostly base on sampling and acquiring digital values of samples by analog-to-digital converters. In this procedure values of sampling points are results of averaging in the aperture time - measurement time. This averaging (or integration) gives reduction of noise but causes systematic errors in estimations of the signal parameters [3]-[5]. In this paper, algorithms for estimation of parameters by signal and zero padding first and then interpolation in the frequency domain are presented.

A sampling process can be modelled with four signals and their frequency spectra in the time and the frequency domain: measured signal $g(t) \stackrel{\mathrm{F}}{\longleftrightarrow} G(f)$ (Figure 1), impulse response of the sampling channel $h(t) \stackrel{F}{\longleftrightarrow} H(f)$ (Figure 2), sampling function $s(t) \stackrel{\mathrm{F}}{\longleftrightarrow} S(f)$ (Figure 3), and the window function of the measurement interval $w(t) \stackrel{F}{\longleftrightarrow} W(f)$ (Figure 4) where $\mathrm{F}$ stands for the Fourier transformation from time domain to frequency domain and vice versa.

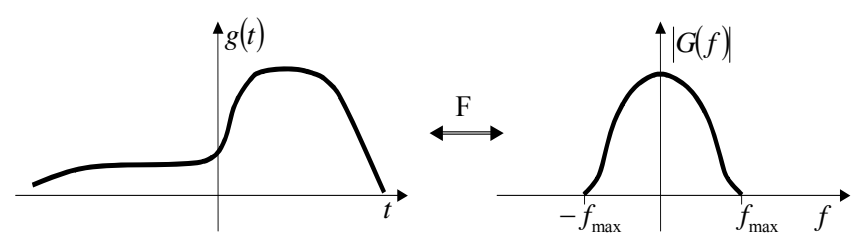

Figure 1. Measured signal.

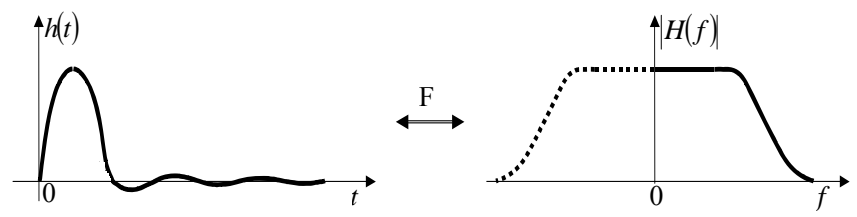

Figure 2. Impulse response of the sampling channel. 


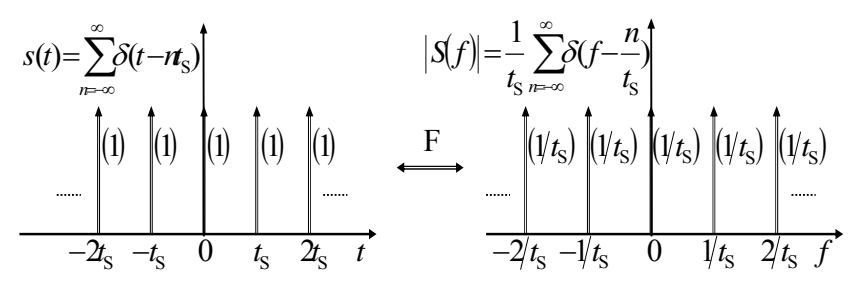

Figure 3. Sampling function in the time and the frequency domain.

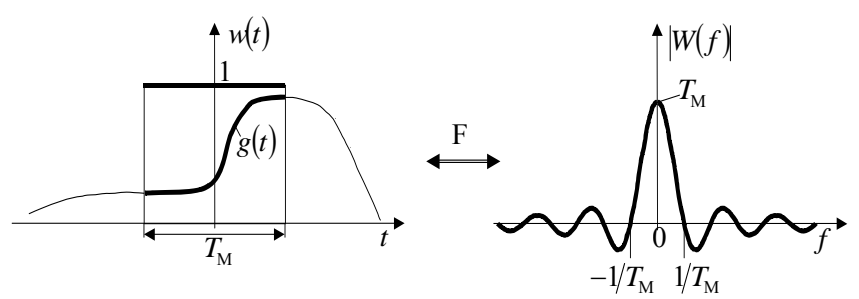

Figure 4. Window function of the measurement interval.

The measured signal $g(t)$ is always filtered by the impulse response of the input front circuit of the measurement channel represented by $h_{f}(t)$ in Figure 5 and after that sampled by the real finit duration sampling pulses represented by $h(t)$ convolved on the sampling function $s(t)$ (Figure 3: typically time uniformly distributed) and finally only a finite number of samples is taken into consideration represented by the window function $w(t)$ (Figure 4) to get a filtered, sampled and windowed signal $g_{f, w}^{*}(t)$ (Figure 5).

Considering that the equivalent of multiplication in the time domain is convolution in the frequency domain $\times \equiv g_{1}(t) \times g_{2}(t) \stackrel{\mathrm{F}}{\longleftrightarrow} \otimes \equiv \int_{-\infty}^{\infty} G_{1}(v) G_{2}(f-v) \mathrm{d} v \quad$ and $\quad$ vice versa $\otimes \equiv \int_{-\infty}^{\infty} g_{1}(\tau) g_{2}(t-\tau) \mathrm{d} \tau \stackrel{\mathrm{F}}{\longleftrightarrow} \times \equiv G_{1}(f) \times G_{2}(f) \quad$ [6] the sampling procedure can be modelled as follows (Figure 5):

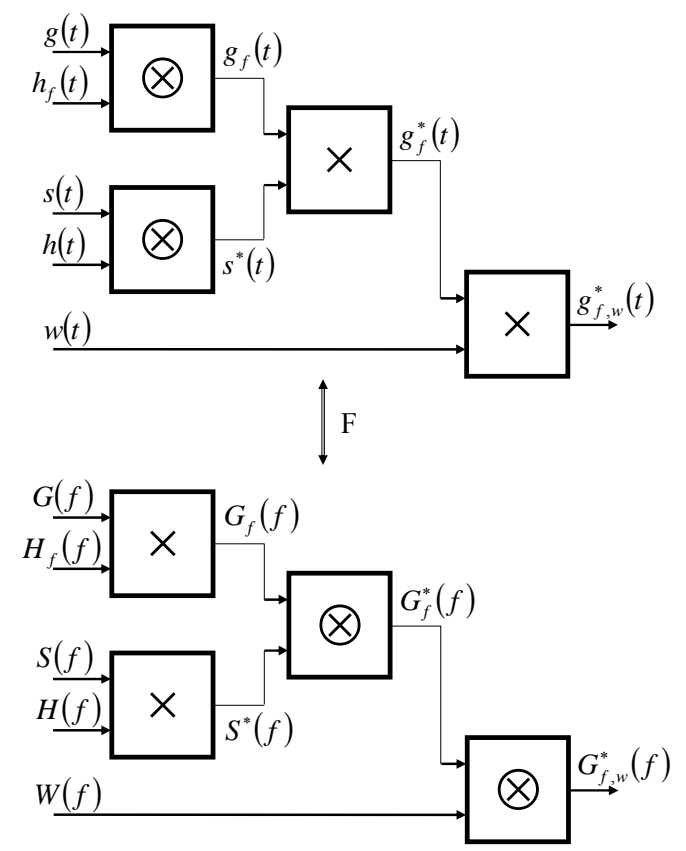

Figure 5. Procedure of sampling in the time and the frequency domain.
It is evident that the spectrum of the sampled signal starts to change with modification of the sampling pulses $h(t) \stackrel{\mathrm{F}}{\longleftrightarrow} H(f)$ (Figures 6 and 7).

\section{PADDING AND ESTIMATION OF PARAMETERS}

Sampling by the frequency $f_{\mathrm{S}}=1 / t_{\mathrm{S}}$ of the periodic band limited signal $g(t)$ composed of $M$ components can be expressed as $w\left(n t_{\mathrm{S}}\right) \cdot \sum_{m=0}^{M-1} A_{m} \sin \left(2 \pi f_{m} n t_{\mathrm{S}}+\varphi_{m}\right)$ with $f_{m}, A_{m}$ and $\varphi_{m}$ as frequency, amplitude, and phase of a particular component, respectively. In the estimation procedure one has to take into account that values of samples (Figure 8: line d) are representatives in the aperture time $t_{\mathrm{ap}}$ or typically average values of the signal in the aperture integration interval (Figure 8: line c). For a demonstration of sampling, in Figure 8 three periods of the one component sine signal are presented with a duty cycle of sampling $D=t_{\text {ap }} / t_{\mathrm{S}}=0.4$ and with sampling ratio of $r=f_{\mathrm{S}} / f_{m}=T_{m} / t_{\mathrm{S}}=1.6$.

With sampling representatives - average values - we lose some information of the signal and especially in the cases where the aperture time is so long that the signal changes significantly

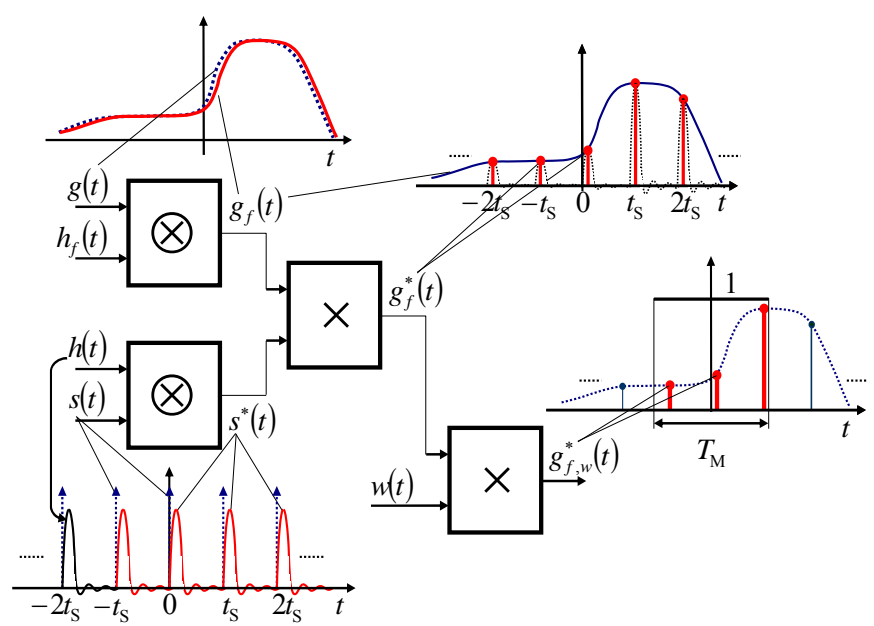

Figure 6 . Signals in the sampling process.

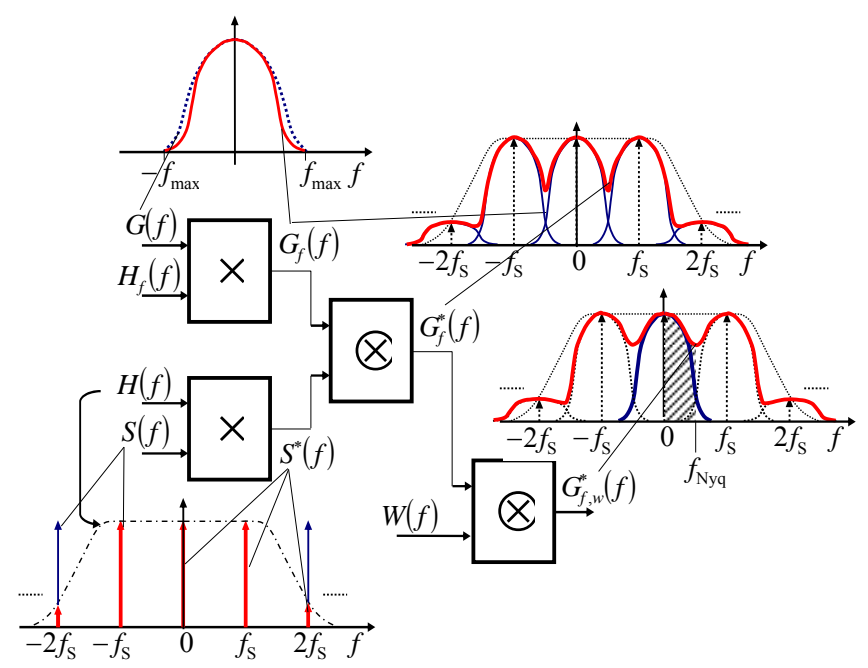

Figure 7. Spectra of signals in the sampling process. 


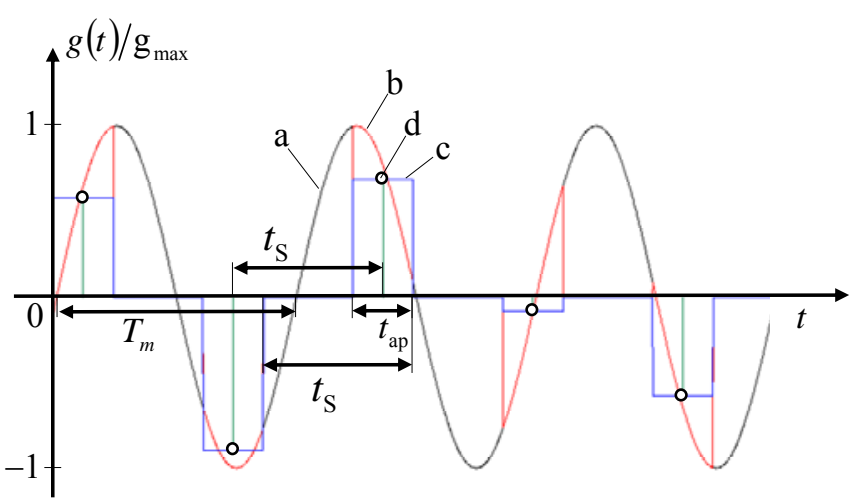

Figure 8. Signals of sampling by averaging in the aperture time: a - original signal, b - truncated signal in the aperture intervals, c - average signal values in the aperture integration interval, $d$ - average value representatives of the sampled signal in the aperture integration interval.

in this interval and where the sampling Nyquist condition is not fulfilled $\left(r=f_{\mathrm{s}} / f_{m}<2\right)$. One possibility to overcome these problems is to add zeroes (known as zero-padding technique [7]) and average values in the aperture times with the duty ratio $D=t_{\text {ap }} / t_{\mathrm{s}}$ (Figure 8: line c). The aperture time is positioned symmetrically around the known sampling instants.

By increasing the number of samples and acquired signal cycles it is possible to detect signals below the Nyquist condition (Figure 9: the whole acquired signal contains around 50 cycles of the sinusoid $\theta_{m}=f_{m} / \Delta f=f_{m} \cdot N t_{\mathrm{s}} \doteq 50$ on $N=20000$ points). An undersampled sine wave still appears as a sampled sine wave but at a lower frequency $f^{\prime}=\left|k f_{\mathrm{S}}-f_{m}\right|$ $(k=1,2, \ldots)$ or expressed by the relative frequency $\theta^{\prime}=\left|k \theta_{\mathrm{S}}-\theta_{m}\right|$ where $\theta_{\mathrm{S}}$ is the relative sampling frequency. With padding points we apparently increase the sampling frequency in relation to the signal frequency.

To estimate parameters of the time-dependent signals containing any periodicity, it is preferable to use a transformation of the signal in the frequency domain. The discrete Fourier transformation (DFT) of the windowed signal $w(k) \cdot g(k)$ on $N$ sampled points at the spectral line $i$ is given by:

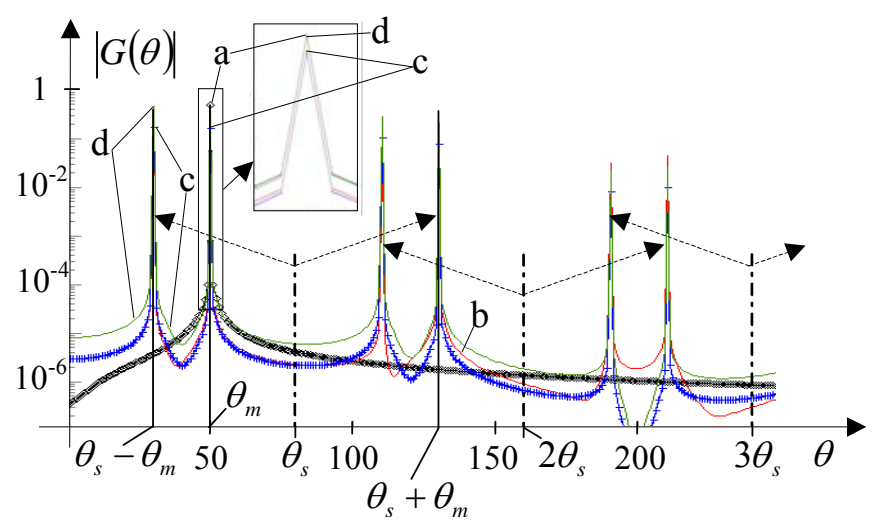

Figure 9. Spectra of signals from Figure 8: a - original signal, b - truncated signal in the aperture intervals, c - signal with average values in the aperture integration interval, $d$ - signal with average values in the aperture integration interval with sinc correction.
$G(i)=-\frac{\mathrm{j}}{2} \sum_{m=0}^{M} A_{m}\left(W\left(i-\theta_{m}\right) \mathrm{e}^{\mathrm{j} \varphi_{m}}-W\left(i+\theta_{m}\right) \mathrm{e}^{-\mathrm{j} \varphi_{m}}\right)$,

where $\theta_{m}=f_{m} / \Delta f=i_{m}+\delta_{m}$ is the frequency component related to the base frequency resolution $\Delta f=1 / T_{\mathrm{M}}=1 / N t_{\mathrm{S}}$ and consists of an integer part and the non-coherent sampling displacement term $-0.5 \leq \delta_{m}<0.5$.

A finite measurement time is a source of dynamic errors, which are shown as leakage parts of the measurement window spectrum convolved on the spectrum of the measured-sampled signal (Figure 9). The long-range leakage contributions can be reduced in more ways: by increasing the measurement time $\Delta f_{\downarrow}=1 / T_{\text {meas }} \uparrow$, by using windows with a faster reduction of the side lobes (like the Rife-Vincent windows class I - RV1, etc. [8]), or by using the multi-point interpolated DFT algorithms. Dedicated algorithms are needed to obtain the correct parameters of the sinusoidal components in the signal. Parameters of the measurement component can be estimated by means of interpolation [9]. From a comparative study [10] it can be concluded that the key for estimating the three basic parameters is in determining the position of the measurement component $\delta_{m}=\theta_{m}-i_{m}$ between the DFT coefficients $G\left(i_{m}\right)$ and $G\left(i_{m}+1\right)$ surrounding the component $m$. In estimations, the well-known expressions for the three-point estimations for frequency (2), amplitude (3), and phase (4) were used. The three-point DFT interpolation gives optimal results owing to: symmetry around the local peak amplitude DFT coefficient; equal suppression of leakage coming from both sides; equal minimal error curves as with one-, five- and multi-point interpolations. Only the order $\mathrm{P}$ of the windows has to be changed using RV1 windows [9].

$$
\begin{aligned}
{ }_{3} \delta_{m} \doteq & (P+1) \cdot \frac{\left|G\left(i_{m}+1\right)\right|-\left|G\left(i_{m}-1\right)\right|}{\left|G\left(i_{m}-1\right)\right|+2\left|G\left(i_{m}\right)\right|+\left|G\left(i_{m}+1\right)\right|} \\
{ }_{3} A_{m} \doteq 2 & \left|\frac{2^{2 P}}{(2 P+2) !} \cdot \frac{\pi \delta_{m}}{\sin \left(\pi \delta_{m}\right)} \cdot \prod_{l=1}^{P+1}\left(l^{2}-\delta_{m}^{2}\right)\right| \\
& \cdot\left(\left|G\left(i_{m}-1\right)\right|+2\left|G\left(i_{m}\right)\right|+\left|G\left(i_{m}+1\right)\right|\right)
\end{aligned}
$$

The single phase can be estimated with the arguments of the three largest local DFT coefficients $\varphi_{i_{m}}=\arg \left[G\left(i_{m}\right)\right]$ [9], [11]:

$$
{ }_{3} \varphi_{m}=\frac{\left(1-\delta_{m}\right) \varphi_{i_{m}-1}+4 \varphi_{i_{m}}+\left(1+\delta_{m}\right) \varphi_{i_{m}+1}}{6}-\frac{2 a \delta_{m}}{3}+\frac{\pi}{2} .
$$

\section{EVALUATION OF THE ESTIMATIONS}

\subsection{Systematic behaviour}

We can estimate three basic parameters of a particular component (true or apparent on Figure 9) by the three-point interpolations since we need only the local largest DFT coefficients. The estimation errors were compared for the frequency (2), amplitude (3), and phase (4) estimations using the Hann window $(P=1)$. The absolute errors of the frequency estimation $|E(\theta)|=\left|\theta_{\text {est. }}-\theta_{\text {true }}\right|$ and the phase estimation $|E(\varphi)|=\left|\varphi_{\text {est. }}-\varphi_{\text {true }}\right|$, and the absolute relative errors of the amplitude estimation $|e(A)|=\left|A_{\text {est. }} / A_{\text {true }}-1\right|$ are first checked for one sine component in the signal with a double scan, varying specific sampling parameters and the phase of the signal 
at particular relative frequencies because the long-range leakages are frequency- and phase-dependent (Figures 10 to 23: $A_{m}=1$, and $\left.-\pi / 2 \leq \varphi \leq \pi / 2, \Delta \varphi=\pi / 18\right)$.

First, the sampling ratio $r=f_{\mathrm{s}} / f_{m}$ was changed to find intervals where the interpolation algorithm can be used (Figures 10 to 19). The absolute maximum error values (from 19 iterations changing phase) at a given sampling ratio are compared using the duty ratio of $D=t_{\text {ap }} / t_{\mathrm{S}}=0.4$. In Figure 10 , we can see that it is possible to estimate the frequency of one component even better than if we have a complete signal also when the sampling ratio $r=f_{\mathrm{s}} / f_{m}$ is in a interval between 1 and 2 (the sampling condition is not fulfilled). The vicinities around the integer values 1 and 2 where we cannot estimate parameters as in the case of the original signal depend on the number of signal cycles in the whole measurement interval $T_{\mathrm{M}}$. The largest value $\theta$ gives a better frequency resolution and borders come closer to integer values 1 and 2 (Figures 10, 11, and 12). The width of the error estimation main-lobe around integer values depends on the position of the investigated component and interspacing between neighbouring components if we have enough sampling points in one period (400 points are used in simulations from analysis in Figures 22 and 23). If we have $\theta=50$ cycles in the measurement interval (Figure 10) we get a basic bin resolution $1 / 50$ and this resolution gives error main-lobe borders $0.85<r<1.15$ and $1.7<r<2.3$ around integers where the frequency estimation errors increase due to leakage influence on the investigated component $\theta_{m}$ from its replicas $k \theta_{\mathrm{S}}-\theta_{m} \quad k=1,2$ (Figures 9

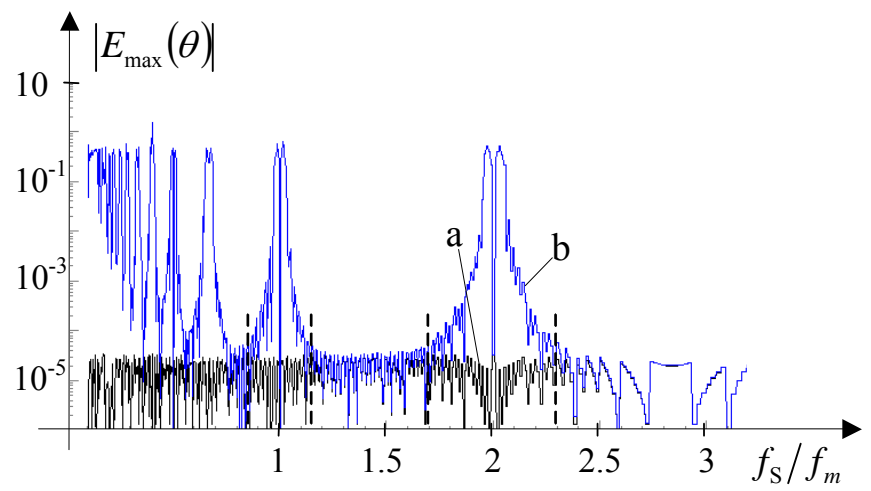

Figure 10. Absolute maximum values of errors of the frequency estimation in relation to the sampling ratio: $\mathrm{a}$ - original signal, $\mathrm{b}$ - signal with average values; $N=20 \mathrm{k}, f_{\mathrm{s}}=5 \mathrm{~Hz} \div 160 \mathrm{~Hz}, \theta_{m} \doteq 50$.

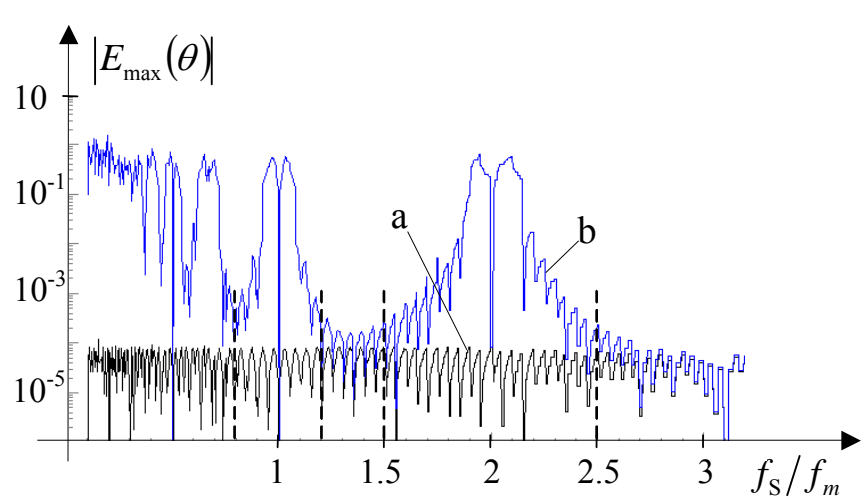

Figure 11. Absolute maximum values of errors of the frequency estimation in relation to the sampling ratio: $\mathrm{a}$ - original signal, $\mathrm{b}$ - signal with average values; $N=8 \mathrm{k}, f_{\mathrm{s}}=2 \mathrm{~Hz} \div 64 \mathrm{~Hz}, \theta_{m} \doteq 20$. and 12).

It can be also noticed that there are error peaks which number increases below $r=2 / 3$ due to replicas $\left|k \theta_{\mathrm{S}}-\theta_{m}\right|$ with higher values of $k=3,4,$. (Figure 12).

Decreasing the bin resolution to $1 / 20$ increases unusable intervals to $0.8<r<1.2$ and $1.5<r<2.5$ (Figure 11). Going in opposite direction by increasing the bin resolution to $1 / 100$ (Figure 12) reduces unusable intervals to $0.9<r<1.1$ and $1.85<r<2.15$, and the frequency can be accurately estimated also in the interval of sampling ratio $1.1<f_{\mathrm{s}} / f_{m}<1.85$ what is below the sampling condition.

In the case of the amplitude estimation (Figures 13 and 15) we need to correct the estimated amplitude or the complete amplitude DFT spectrum (Figure 9, line d) by the well-known sinc correction $k_{\text {corr-sinc }}=\left(\pi \cdot t_{\text {ap }} / T_{m}\right) / \sin \left(\pi \cdot t_{\text {ap }} / T_{m}\right)$ [3]. We see the same behaviour of the errors in the cases of amplitude and phase estimations as with the frequency estimation (Figures 13 to 16). A bin resolution of $1 / 50$ reduces unusable intervals $0.85<r<1.15$ and $1.8<r<2.2$ for the phase estimation as for frequency estimation (Figure 14) and even better for the amplitude estimation (Figure 13: unusable intervals $0.9<r<1.1$ and $1.7<r<2.3)$. A resolution of $1 / 100$ further reduces unusable intervals to $0.92<r<1.08$ and $1.85<r<2.15$ (Figures 15 and 16).

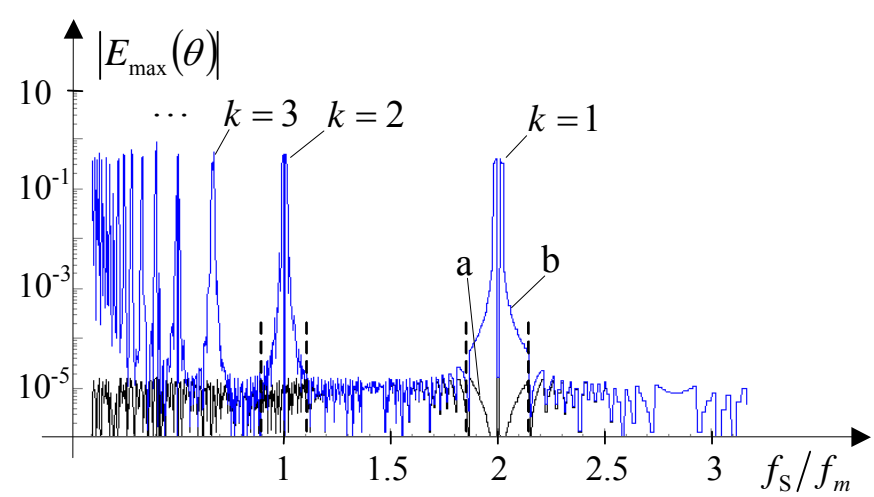

Figure 12. Absolute maximum values of errors of the frequency estimation in relation to the sampling ratio: $\mathrm{a}$ - original signal, $\mathrm{b}$ - signal with average values; $N=40 \mathrm{k}, f_{\mathrm{s}}=10 \mathrm{~Hz} \div 320 \mathrm{~Hz}, \theta_{m} \doteq 100$.

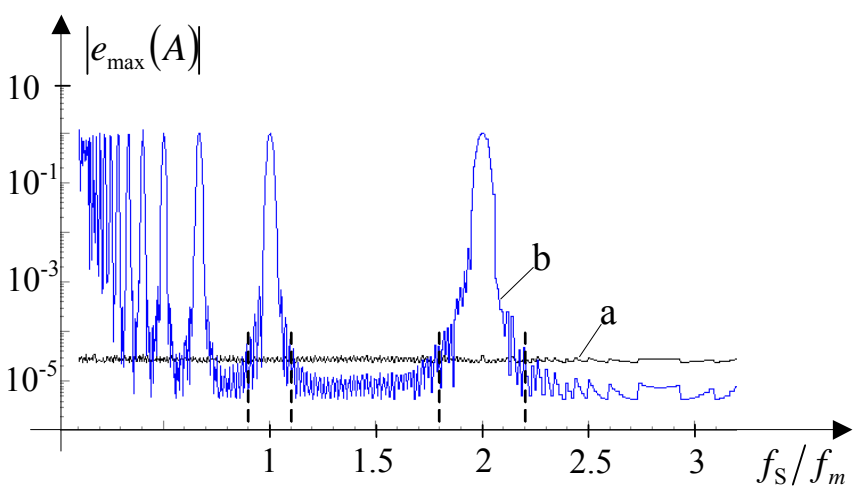

Figure 13. Absolute maximum values of relative errors of the amplitude estimation in relation to the sampling ratio: $a-$ original signal, $b-$ signal with average values; $N=20 \mathrm{k}, f_{\mathrm{S}}=5 \mathrm{~Hz} \div 160 \mathrm{~Hz}, \theta_{m} \doteq 50$. 


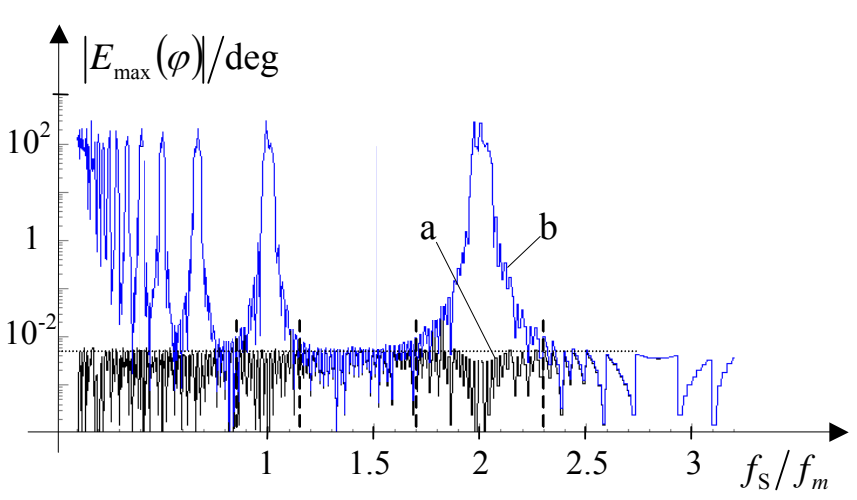

Figure 14. Absolute maximum values of errors of the phase estimation in relation to the sampling ratio: $a$ - original signal, $\mathrm{b}$ - signal with average values; $N=20 \mathrm{k}, f_{\mathrm{s}}=5 \mathrm{~Hz} \div 160 \mathrm{~Hz}, \theta_{m} \doteq 50$.

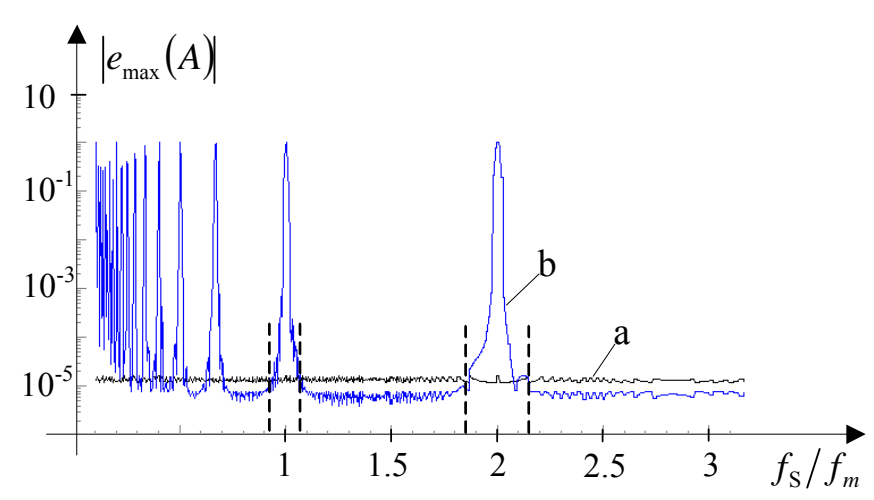

Figure 15. Absolute maximum values of relative errors of the amplitude estimation in relation to the sampling ratio: $a-$ original signal, $b-$ signal with average values; $N=40 \mathrm{k}, f_{\mathrm{s}}=10 \mathrm{~Hz} \div 320 \mathrm{~Hz}, \theta_{m} \doteq 100$.

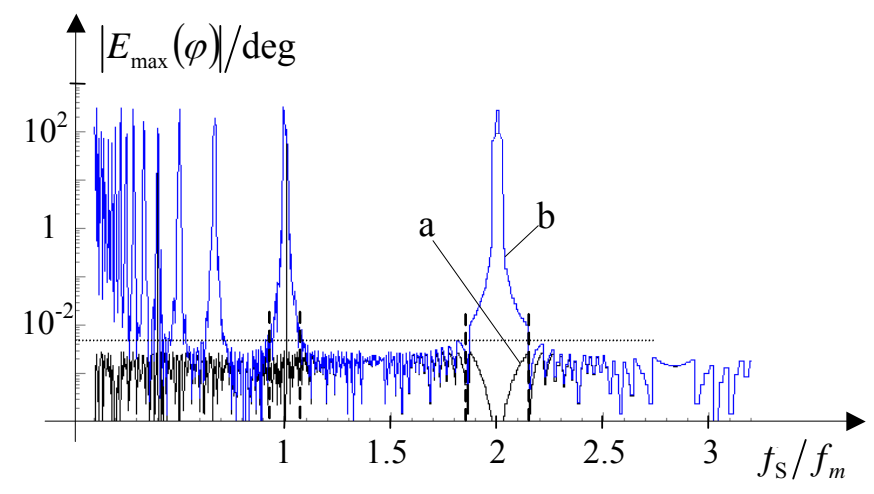

Figure 16. Absolute maximum values of errors of the phase estimation in relation to the sampling ratio: $\mathrm{a}$ - original signal, $\mathrm{b}$ - signal with average values; $N=40 \mathrm{k}, f_{\mathrm{S}}=10 \mathrm{~Hz} \div 320 \mathrm{~Hz}, \theta_{m} \doteq 100$.

Algorithms were analysed also by the multi-component signal. The second harmonic component as the closest and the most disturbing component was added with amplitude $A_{2}=A_{1} / 10(T H D=0.1)$ and phase $\varphi_{2}=0$, other parameters of simulations are the same as for Figure 8. We get the most disturbing replicas of the second harmonic at positions $r=1.5$ and 3 due to the leakage effect $f^{\prime}=\left|k f_{\mathrm{S}}-2 f_{m}\right| \quad(k=1,2)$ (Figures 17 to 19). Simulation results show that the proposed estimation procedures give very good results when the sampling

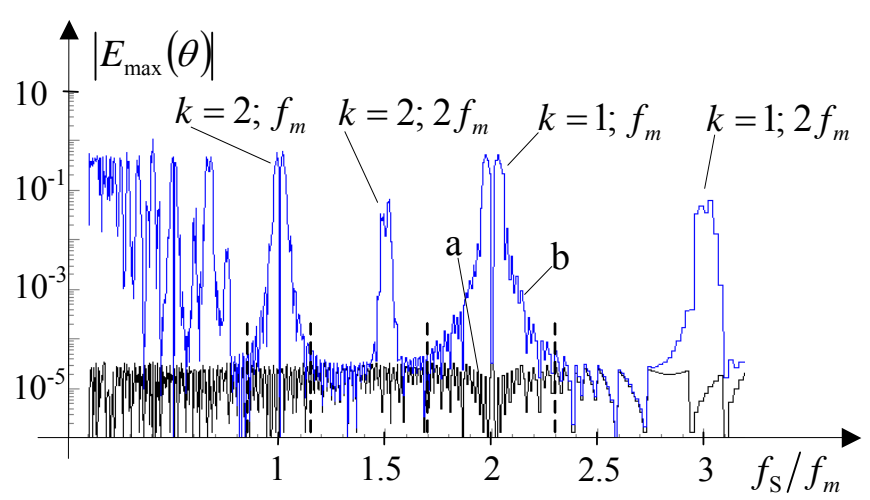

Figure 17. Absolute maximum values of errors of the frequency estimation in relation to the sampling ratio: $a$ - original signal, $b$ - signal with average values; $N=20 \mathrm{k}, f_{\mathrm{S}}=5 \mathrm{~Hz} \div 160 \mathrm{~Hz}, \theta_{m} \doteq 50$.

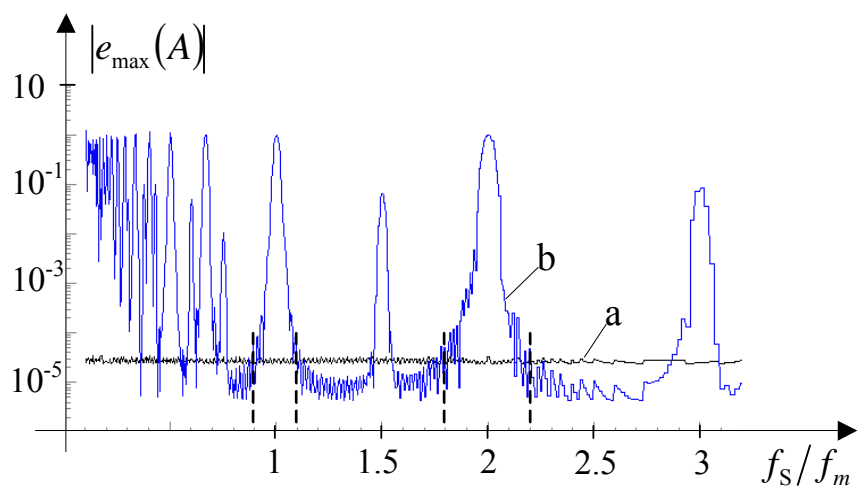

Figure 18. Absolute maximum values of relative errors of the amplitude estimation in relation to the sampling ratio: $a-$ original signal, $b-$ signal with average values; $N=20 \mathrm{k}, f_{\mathrm{S}}=5 \mathrm{~Hz} \div 160 \mathrm{~Hz}, \theta_{m} \doteq 50$.

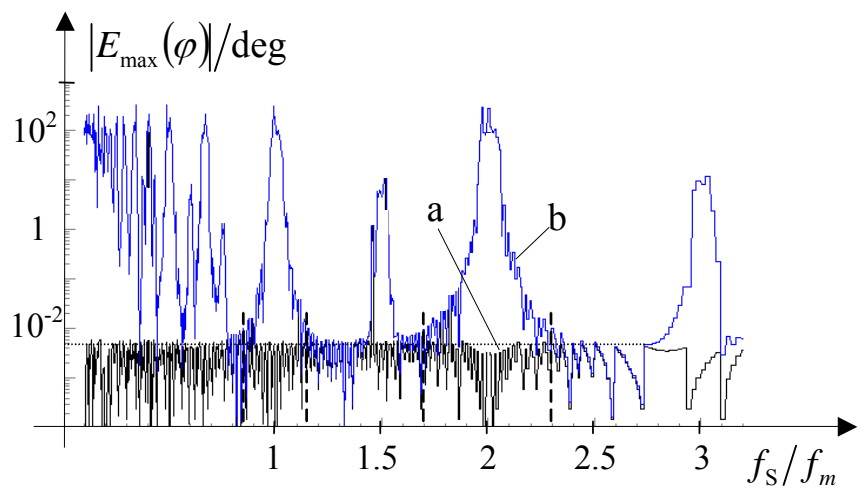

Figure 19. Absolute maximum values of errors of the phase estimation in relation to the sampling ratio: $a$ - original signal, $b$ - signal with average values; $N=20 \mathrm{k}, f_{\mathrm{s}}=5 \mathrm{~Hz} \div 160 \mathrm{~Hz}, \theta_{m} \doteq 50$.

condition is fulfilled, but beyond the Nyquist frequency the THD has to be low $(<0.01)$.

If we change the duty ratio $D=t_{\text {ap }} / t_{\mathrm{s}}$ in the sampling interval (changing the aperture time at a fixed sampling frequency) the estimation errors do not change very much in comparison to the estimation of the original signal if we correct the estimation by a sinc correction [12]. In Figures 20 and 21 the duty ratio was changed almost in the whole possible interval $D=0.001 \div 0.998 \quad$ at $r=f_{\mathrm{S}} / f_{m}=1.6 \quad$ with $\quad N_{\mathrm{P}}=400$ 


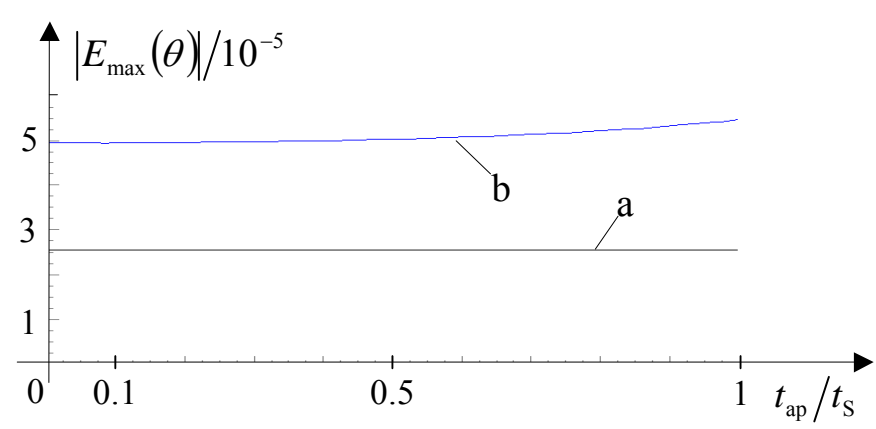

Figure 20. Absolute maximum values of errors of the frequency estimation in relation to the duty ratio: a - original signal, $b$ - signal with average values in the aperture interval.

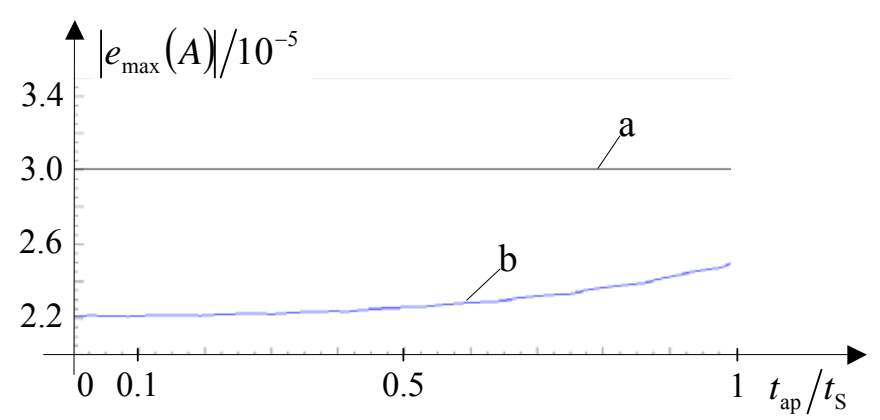

Figure 21. Absolute maximum values of relative errors of the amplitude estimation in relation to the duty ratio: a - original signal, b - signal with average values in the aperture integration interval with sinc correction.

samples in one period (other parameters were the same as in Figure 8).

The estimation also much depends on the number of points in the period $N_{P}$ and in the whole measurement interval. Padding with more points (average signal values and zero values) will improve estimations since interpolation equations (2), (3), and (4) are derived for large number of points $N>>1$ [9]. In Figures 22 and 23, the number of points in the period $N_{P}$ was changed from $N_{\mathrm{P}}=10$ to more than $N_{\mathrm{P}}=512$ (other parameters were the same as in Figure 8). We can see that the frequency estimation does not differ significantly if we have reduced the information of the signal (Figure 22: curves a and b) and both estimations errors decrease with increasing number of points.

The amplitude estimation much more depends on the number of points (Figure 23) but after having more than $N_{\text {P }}=128$ points per period the estimation errors drop to the level as with the estimation of the original signal without averaging in the aperture time.

\subsection{Noise propagation}

The price for the effective leakage reduction is in the increase of the estimation uncertainties related to the unbiased Cramér-Rao bounds [13] fixed by the signal-to-noise-ratio for a particular component $S N R_{m}=A_{m}^{2} /\left(2 \sigma_{t}^{2}\right)$ corrupted by a white noise with standard uncertainty $\sigma_{t}$ [14]. In Figures 24 and 26, there are standard uncertainties of the frequency, amplitude, and phase estimations related to the CR bounds, respectively.

$\sigma_{\theta} \geq \frac{\sqrt{3}}{\pi} \frac{1}{\sqrt{S N R}} \frac{1}{\sqrt{N}}=\sigma_{\mathrm{CRB}, \theta}$,

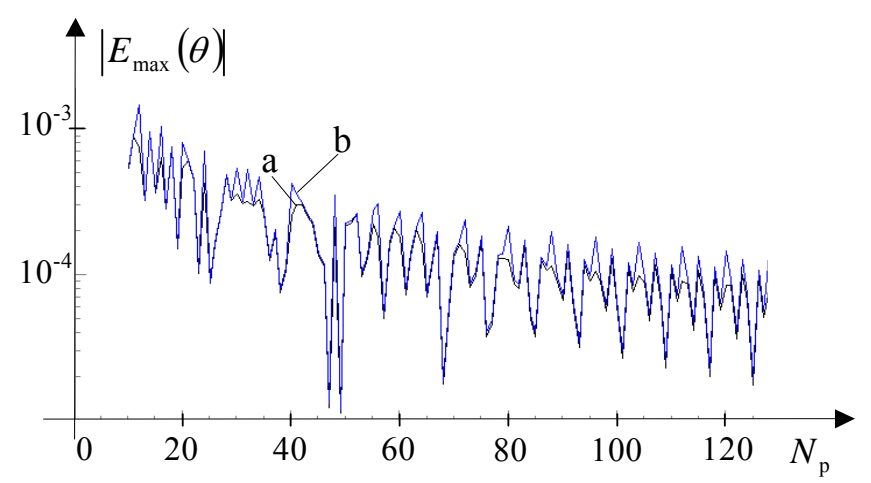

Figure 22. Absolute maximum values of errors of the frequency estimation in relation to the number of sampling points in the period: a - original signal, $b$ - signal with average values in the aperture interval.

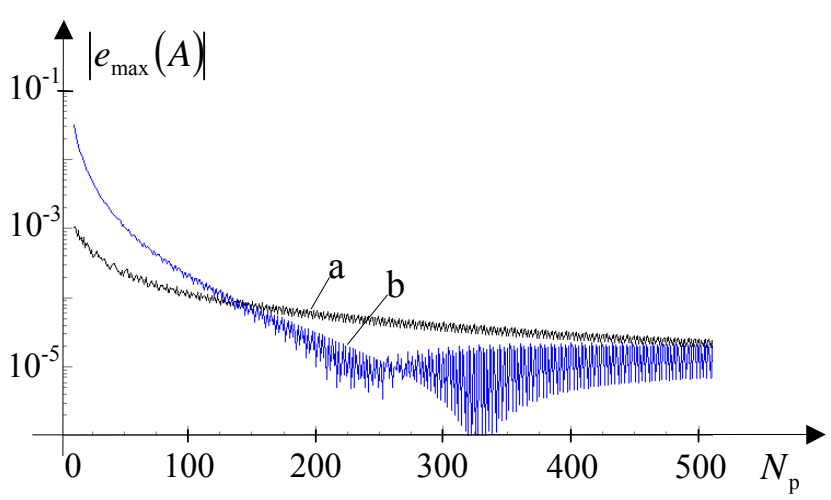

Figure 23. Absolute maximum values of relative errors of the amplitude estimation in relation to the number of sampling points in the period: a original signal, $b$ - signal with average values in the aperture integration interval with sinc correction.

$$
\begin{aligned}
& \sigma_{A} \geq \frac{1}{\sqrt{S N R}} \frac{1}{\sqrt{N}}=\sigma_{\mathrm{CRB}, A}, \\
& \sigma_{\varphi} \geq 2 \frac{1}{\sqrt{S N R}} \frac{1}{\sqrt{N}}=\sigma_{\mathrm{CRB}, \varphi} .
\end{aligned}
$$

Moving away from integers of the relative frequency, what is the case when the sampling ratio is below 2, the standard uncertainties increase in relation to the minimal attainable values (Figure 24 for the frequency estimation, Figure 25 for the amplitude estimation, and Figure 26 for the phase estimation) but these changes can be neglected. In the case of amplitude estimation the standard deviations even decrease if the frequency $\theta$ is estimated first.

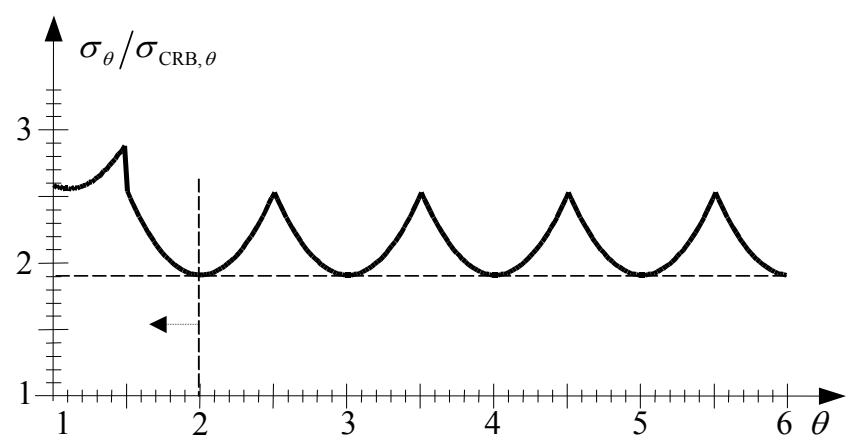

Figure 24. Standard uncertainty of the three-point displacement estimation (2) related to the CR bound (5). 


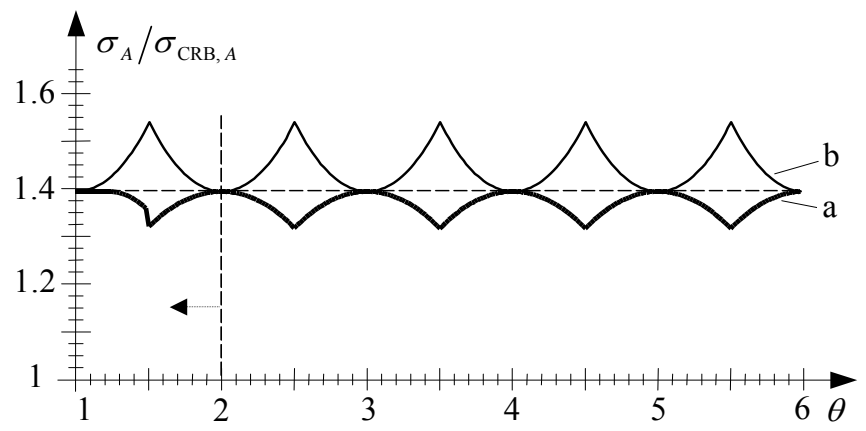

Figure 25. Standard uncertainty of the amplitude three-point estimation (3) related to the CRB (6); $\mathrm{a}-\theta$ is estimated, $\mathrm{b}-\theta$ is known.

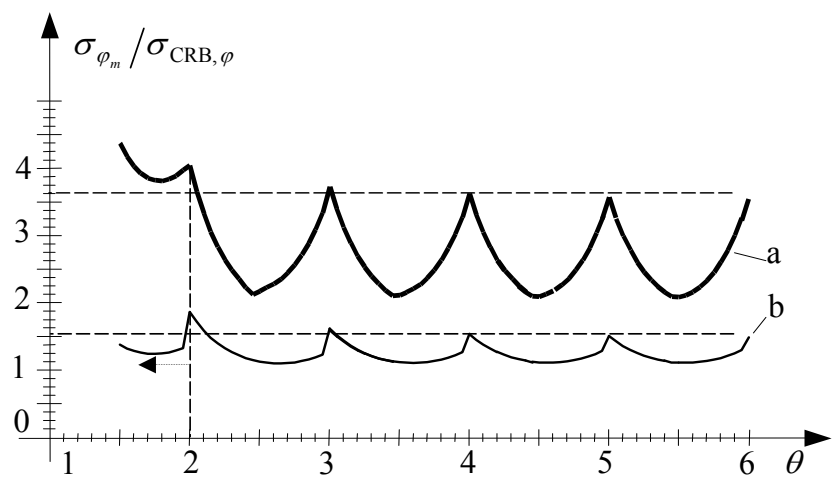

Figure 26. Ratios of the uncertainties of the phase three-point estimation (4) related the CRB (7); $\mathrm{a}-\theta$ is estimated, $\mathrm{b}-\theta$ is known.

\section{EXPERIMENTAL RESULTS}

To demonstrate the proposed algorithms in reducing both estimation errors (phase and noise contributions at different frequencies) in a real measurement environment we use a digitizing voltmeter to acquire signal, Agilent 3458A [15] and a stable voltage generator, Keysight $33500 \mathrm{~B}$ [16] to generate a nominal sine voltage with amplitude $A_{u, \mathrm{n}}=1 \mathrm{~V}$ $(T H D<0.04 \%)$ and changing frequency. In opposite to simulations, the sampling frequency was set at $f_{\mathrm{S}}=10 \mathrm{kHz}$ $\rightarrow t_{\mathrm{S}}=100 \mu \mathrm{s}$ and the signal frequency was changed from $f_{\mathrm{m}}=100 \mathrm{kHz}$ down to $f_{\mathrm{m}}=3.125 \mathrm{kHz}$ to achieve the same sampling ratio $r=f_{\mathrm{s}} / f_{\mathrm{m}}=0.1 \div 3.2$. The fixed aperture time $t_{\text {ap }}=40 \mu \mathrm{s}$ determined the duty cycle of sampling $D=t_{\text {ap }} / t_{\mathrm{S}}=0.4$ and with $n_{\text {s.p. }}=80$ acquired sampling points the measurement time was $T_{\mathrm{M}}=n_{\mathrm{s} . \mathrm{p}} t_{\mathrm{s}}=8 \mathrm{~ms}$ with a frequency resolution of $\Delta f=1 / T_{\mathrm{M}}=125 \mathrm{~Hz}$. As in the simulations, $N_{\text {padd }}=400$ padding points (for signal and zero padding) was used around the acquired points, and altogether $N=n_{\text {s.p. }} \cdot N_{\text {padd }}=32 \mathrm{k}$ was used in the interpolation DFT algorithms. Each sampling sequence of 80 points was synchronized using the 'sync out' terminal of the generator and the external trigger input of the voltmeter.

Maximal estimation errors are shown in Figures 27 to 29, where reference values were those set on the generator $(A=1$, $f=100 \mathrm{kHz} \div 3.125 \mathrm{kHz}$ and $-\pi / 2 \leq \varphi \leq \pi / 2, \Delta \varphi=\pi / 18)$

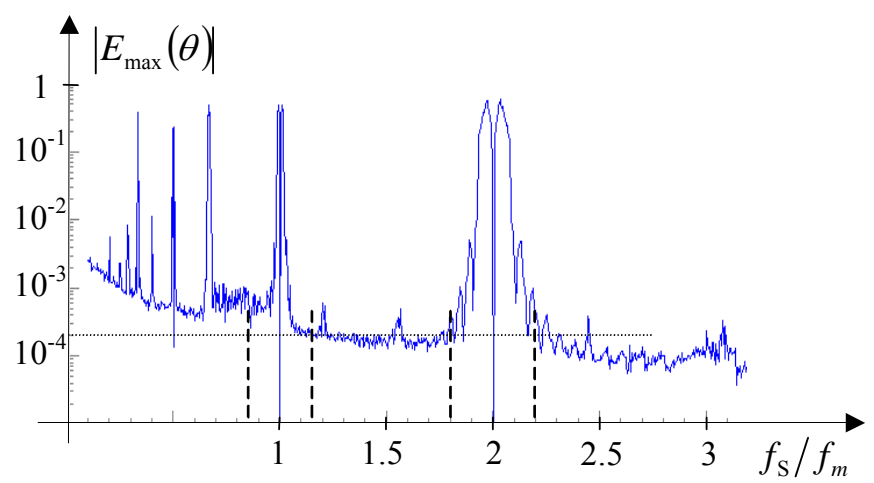

Figure 27. Absolute maximum values of errors of the frequency estimation in relation to the sampling ratio; $n_{\text {s.p. }}=80, N=32 \mathrm{k}, f_{\mathrm{S}}=10 \mathrm{kHz}$, $\theta_{\mathrm{m}}=f_{\mathrm{m}} / \Delta f=800 \div 25$.

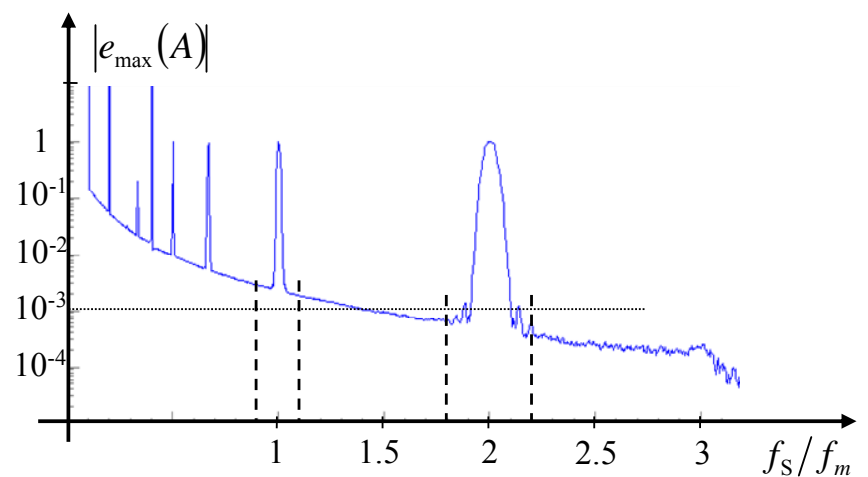

Figure 28. Absolute maximum values of the relative errors of the amplitude estimation in relation to the sampling ratio; $n_{\text {s.p. }}=80, N=32 \mathrm{k}$, $f_{\mathrm{s}}=10 \mathrm{kHz}, \theta_{\mathrm{m}}=f_{\mathrm{m}} / \Delta f=800 \div 25$.

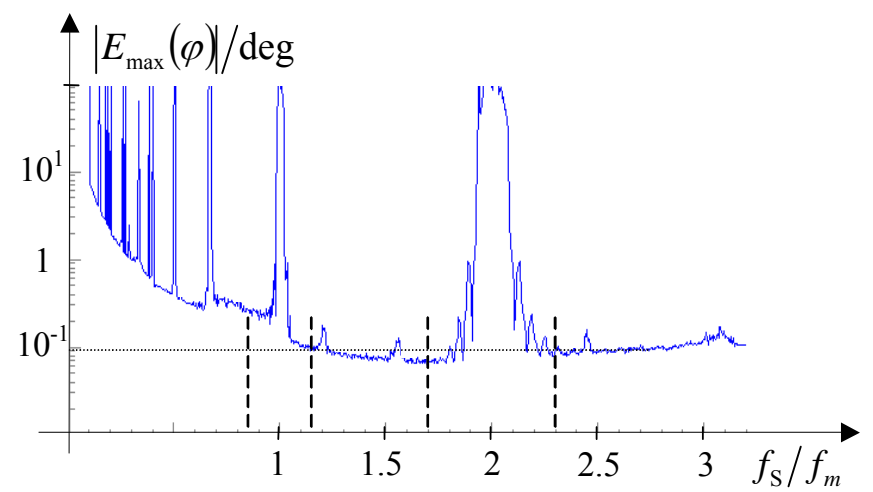

Figure 29. Absolute maximum values of errors of the phase estimation in relation to the sampling ratio; $n_{\text {s.p. }}=80, N=32 \mathrm{k}, f_{\mathrm{s}}=10 \mathrm{kHz}$, $\theta_{\mathrm{m}}=f_{\mathrm{m}} / \Delta f=800 \div 25$.

and with 50 trials at each frequency and phase. The estimation error behaviours are very close to those in the simulations.

It can be noticed that systematic error contributions behaves as expected and a very small second harmonic component is presented in the measurement system. The frequency estimation (Figure 27) can be compared with the simulation results from Figure 10 except the noise floor is higher and at the level of $E_{\max }(\theta) \approx 2 \cdot 10^{-4}$ even in the interval below the sampling condition $1.15<f_{\mathrm{s}} / f_{m}<1.8$. The results of the 
amplitude (Figure 28) and the phase estimations (Figure 29) are worse in comparison to the simulation results (Figures 13 and 14) due to inaccurate values at the output of the generator and inaccuracy of the sampling voltmeter, but the systematic contributions of errorss confirm the expected behaviour like with the frequency estimation.

\section{CONCLUSIONS}

The paper proposes algorithms for the estimation of basic sinusoidal parameters (frequency, amplitude, and phase of the frequency component), when the acquired sampling points do not fulfil the sampling condition and some signal information is lost due to signal averaging in the aperture time. In the proposed procedure, the empty space between successive real sampling points is virtually padded by average values of the real sampling point in the interval of knowing aperture and zeroes in the rest of the sampling interval. This procedure with suitable large acquired cycles in the whole measurement interval improves the frequency resolution, and the interpolated DFT estimation algorithms can be adopted for particular frequency components.

In many cases the number of sampling points is limited but by performing the algorithms on a computer we can increase padding points and with this nearing the errors to the level as with estimation on the theoretically original signal without averaging in the aperture time and with the number of points equal to all padding points. Simulation and experimental results show that the parameters' estimations are possible also even in the interval below the sampling condition.

\section{REFERENCES}

[1] G. D’Antona, A. Ferrero, Digital Signal Processing for Measurement Systems. Theory and Applications, Springer Science, 2006.

[2] D. Agrež, "Dynamics of frequency estimation in the frequency domain", IEEE Trans. Instr. Meas. 56 (2007) pp. 2111-2118.
[3] R. L. Swerlein, "A $10 \mathrm{ppm}$ accurate digital AC measurement algorithm", Proc. NCSL Workshop Symp., 1991, pp. 17-36.

[4] G. A. Kyriazis, "Extension of Swerlein's Algorithm for AC Voltage Measurement in the Frequency Domain", IEEE Trans. Instr. Meas. 52 (2003) pp. 367-370.

[5] H. E. van den Brom, E. Houtzager, S. Verhoeckx, Q. E. V. N. Martina, G. Rietveld, "Influence of Sampling Voltmeter Parameters on RMS Measurements of Josephson StepwiseApproximated Sine Waves", IEEE Trans. Instr. Meas. 58 (2009) pp. 3806-3812.

[6] W. McC. Siebert, Circuits, Signals and Systems, The MIT Press, McGraw-Hill, Cambridge, New York, 1986, pp. 497-502.

[7] G. Leus, M. Moonen, "Semi-blind channel estimation for block transmissions with non-zero padding", Records of Conf. on Signals, Systems and Comp., 2001, pp. 762-766.

[8] F. J. Harris: "On the use of windows for harmonic analysis with the discrete Fourier transform", Proc. IEEE 66 (1978) pp. 51-83.

[9] J. Štremfelj, D. Agrež, "Nonparametric estimation of power quantities in the frequency domain using Rife-Vincent windows", IEEE Trans. Instr. Meas. 62 (2013) pp. 2171-2184.

[10] J. Schoukens, R. Pintelon, H. Van Hamme, "The interpolated fast Fourier transform: A comparative study", IEEE Trans. Instr. Meas. 41 (1992) pp. 226-232.

[11] T. J. Stewart, "Spectral leakage errors when using an Agilent $3458 \mathrm{~A}$ to measure phase at mains power frequencies", Proc. NCSL Workshop Symp. 1991, pp. 17-36.

[12] D. Agrež, D. Ilić, J. Drnovšek, "Estimation of periodic signal parameters by signal and zero padding", in Proc. XXI IMEKO World Congress/2015, Prague, Czech Republic, 2015, TC4: 474479.

[13] A. Moschitta, P. Carbone, "Cramer-Rao lower bound for parametric estimation of quantized sinewaves", Proc. IEEE IMTC/2004, Como, Italy, 2004, pp. 1724-1729.

[14] Widrow B, Kollar I, Quantization Noise, Cambridge Univ. Press, 2008.

[15] 3458A Multimeter - User's Guide, Ed. 5, 1988-2012, 2011, Agilent Technologies.

[16] 33500B Series Waveform Generators - Data Sheet, 59910692EN, 2015, Keysight Technologies. 